\title{
A Zero-Test and an Interpolation Algorithm for the Shifted Sparse Polynomials
}

\author{
Dima Grigoriev ${ }^{\star 1}$ and Marek Karpinski ${ }^{\star 2}$ \\ 1 Dept. of Computer Science, the Pennsylvania State University, University Park, \\ PA 16802 \\ 2 Dept. of Computer Science, University of Bonn, 5300 Bonn 1, and International \\ Computer Science Institute, Berkeley, California
}

\begin{abstract}
Recall that a polynomial $f \in F\left[X_{1}, \ldots, X_{n}\right]$ is $t$-sparse, if $f=\sum \alpha_{I} X^{I}$ contains at most $t$ terms. In [BT 88], [GKS 90] (see also [GK 87 ] and [ $\mathrm{Ka} 89]$ ) the problem of interpolation of $t$-sparse polynomial given by a black-box for its evaluation has been solved. In this paper we shall assume that $F$ is a field of characteristic zero. One can consider a $t$ sparse polynomial as a polynomial represented by a straight-line program or an arithmetic circuit of the depth 2 where on the first level there are multiplications with unbounded fan-in and on the second level there is an addition with fan-in $t$.

In the present paper we consider a generalization of the notion of sparsity, namely we say that a polynomial $g\left(X_{1}, \ldots, X_{n}\right) \in F\left[X_{1}, \ldots, X_{n}\right]$ is shifted t-sparse if for a suitable nonsingular $n \times n$ matrix $A$ and a vector $B$ the polynomial $g\left(A\left(X_{1}, \ldots, X_{n}\right)^{T}+B\right)$ is $t$-sparse. One could consider $g$ as being represented by a straight-line program of the depth 3 where on the first level (with the fan-in $n+1$ ) a linear transformation $A\left(X_{1}, \ldots, X_{n}\right)^{T}+B$ is computed. One could also consider a shifted $t$-sparse polynomial as $t$-sparse with respect to other coordinates $\left(Y_{1}, \ldots, Y_{n}\right)^{T}=A\left(X_{1}, \ldots, X_{n}\right)^{T}+B$.

We assume that a shifted $t$-sparse polynomial $g$ is given by a black-box and the problem we consider is to construct a transformation $A\left(X_{1}, \ldots, X_{n}\right)^{T}+B$. As the complexity of the designed below algorithm (see the Theorem in which we describe the variety of all possible $A, B$ and the corresponding $t$-sparse representations of $\left.g\left(A\left(X_{1}, \ldots, X_{n}\right)^{T}+B\right)\right)$ depends on $d^{n^{4}}$ where $d$ is the degree of $g$, we could first interpolate $g$ within time $d^{O(n)}$ and suppose that $g$ is given explicitly. It would be interesting to get rid of $d$ in the complexity bounds as it is usually done in the interpolation of sparse polynomials ([BT 88], [GKS 90], [Ka 89]). The main technical tool we rely on is the criterium of $t$-sparsity based on Wronskian ([GKS 91], [GKS 92]), the latter criterium has a parametrical nature (so we can select $t$-sparse polynomials from a given parametrical family of polynomials) unlike the approach in [BT 88] using BCH-codes.
\end{abstract}

\footnotetext{
* Work partially done while visiting the Dept. of Computer Science, University of Bonn. On leave from the Steklov Mathematical Institute, Fontanka 27, St. Petersburg, 191011 Russia

** Supported in part by Leibniz Center for Research in Computer Science, by the DFG Grant KA 673/4-1 and by the SERC Grant GR-E 68297
} 
We could directly consider (see the Theorem) the multivariate polynomials (section 3), but to make the exposition clearer before that we first study (see the proposition) the one-variable case (section 2). First at all we recall (section 1) the criterium of $t$-sparsity and based on it interpolation method for $t$-sparse multivariable polynomials.

In the last section 4 we design a zero-test algorithm for shifted $t$-sparse polynomials with the complexity independent on $d$.

\section{A Criterium of $t$-sparsity and the Interpolation}

Let $p_{1}, \ldots, p_{n}$ be pairwise distinct primes and denote by $D$ a linear operator mapping $D: X_{1} \rightarrow p_{1} X_{1}, \ldots, D: X_{n} \rightarrow p_{n} X_{n}$. We recall a criterium of $t$ sparsity (cf. also [BT 88]).

Lemma 1. ([GKS 91], [GKS 92]) A polynomial $f \in F\left[X_{1}, \ldots, X_{n}\right]$ is t-sparse if and only if the Wronskian

$$
W_{f}\left(X_{1}, \ldots, X_{n}\right)=\operatorname{det}\left(\begin{array}{llll}
f & D f & \ldots & D^{t} f \\
D f & D^{2} f & \ldots & D^{t+1} f \\
\vdots & \vdots & & \vdots \\
D^{t} f & D^{t+1} f & \ldots & D^{2 t} f
\end{array}\right) \in F\left[X_{1}, \ldots, X_{n}\right]
$$

vanishes identically.

An interpolation method from [BT 88] (see also [KY 88]) actually considers the Wronskian $W_{f}(1, \ldots, 1)$ at the point $(1, \ldots, 1)$ and is based on the following Lemma 2. ([BT 88]) If $f$ is exactly t-sparse (i.e., $f$ contains exactly t terms), then the reduced Wronskian does not vanish $\bar{W}_{f}(1, \ldots, 1)=\operatorname{det}\left(\begin{array}{lll}f(1, \ldots, 1) & (D f)(1, \ldots, 1) & \ldots\left(D^{t-1} f\right)(1, \ldots, 1) \\ \vdots & \vdots & \vdots \\ \left(D^{t-1} f\right)(1, \ldots, 1) & \left(D^{t} f\right)(1, \ldots, 1) \ldots\left(D^{2 t-2} f\right)(1, \ldots, 1)\end{array}\right) \neq 0$

at the point $(1, \ldots, 1)$.

Thus, if $f=\sum \alpha_{I} X^{I}$ is exactly $t$-sparse and if a (characteristic) polynomial $\chi(Z)=\sum_{0 \leq j \leq t} \gamma_{j} Z^{j} \in \mathbb{Z}[Z]$ has as its $t$ roots $p^{I}$ for all exponent vectors $I$ occuring in $f$ (where for $I=\left(i_{1}, \ldots, i_{n}\right.$ ) we denote $p^{I}=p_{1}^{i_{1}} \cdots p_{n}^{i_{n}}$ ), then $\sum_{0 \leq j \leq t} \gamma_{j} D^{j} f=0$ and hence

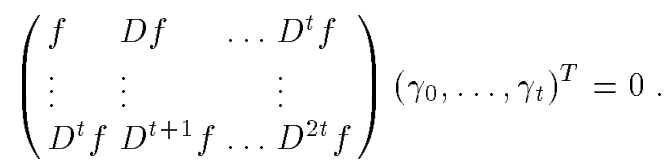

Therefore, a linear system

$$
\left(\begin{array}{lll}
f(1, \ldots, 1) & (D f)(1, \ldots, 1) & \ldots\left(D^{t} f\right)(1, \ldots, 1) \\
\vdots & \vdots & \vdots \\
\left(D^{t} f\right)(1, \ldots, 1) & \left(D^{t+1} f\right)(1, \ldots, 1) & \ldots \\
\left(D^{2 t} f\right)(1, \ldots, 1)
\end{array}\right)\left(Y_{0}, \ldots, Y_{t}\right)^{T}=o
$$

has (up to a constant multiple) a unique (by lemma 2$)$ solution $\left(Y_{0}, \ldots, Y_{t}\right)=$ $\left(\gamma_{0}, \ldots, \gamma_{t}\right)$ which gives the coefficients of $\chi$, thereby its roots $p^{I}$ and finally $I$. 


\section{One-variable Shifted Sparse Polynomials}

A polynomial $g \in F[X]$ is called shifted $t$-sparse if for an appropriate $b$ a polynomial $g(X-b)$ is $t$-sparse (so the origin is shifted from 0 to $b$ ). If $t$ is the least possible, we say that $g$ is minimally shifted $t$-sparse, this notion relates also to the multivariable case. Let $F=\mathbb{Q}$. Usually we take $b$ from the algebraic closure $\overline{\mathbb{Q}}$ (we could also consider $b$ from $\mathbb{R}$ ). Assume that the bit-size of the (rational) coefficients of $g$ does not exceed $M$.

Consider a new variable $Y$ and an $\mathbb{Q}(Y)$-linear transformation of the ring $\mathbb{Q}(Y)[X]$ mapping $D_{1}: X \rightarrow p_{1} X+\left(p_{1}-1\right) Y$. Denote

$$
\mathcal{W}_{g}(X, Y)=\operatorname{det}\left(\begin{array}{llll}
g & D_{1} g & \ldots & D_{1}^{t} g \\
\vdots & \vdots & \vdots \\
D_{1}^{t} g & D_{1}^{t+1} g & \ldots & D_{1}^{2 t} g
\end{array}\right) \in \mathbb{Q}[X, Y]
$$

Lemma 3. $g$ is shifted t-sparse if and only if for some $Y=b$ a polynomial $\mathcal{W}_{g}(X, b)$ vanishes identically. Moreover in this case a polynomial $g(X-b)$ is t-sparse.

Proof. If $g(X-b)$ is $t$-sparse, then the expansion $g=\sum_{j} \beta_{j}(X+b)^{j}$ into the powers of $(X+b)$ contains at most $t$ terms. Lemma 1 implies that $\mathcal{W}_{g}(X, b)$ vanishes identically. The other direction follows also from lemma 1 which completes the proof.

Observe that for almost every $b$ the polynomial $g(X-b)$ has exactly $(d+1)$ terms, where $d=\operatorname{deg}(g)$, since in the polynomial $g(X-Y) \in \mathbb{Q}[X, Y]$ the coefficient in the power $X^{S}$ is a polynomial in $Y$ of degree exactly $d-S, 0 \leq$ $S<d$.

Lemma 3 provides an algorithm for finding $t$ such that $g$ is minimal shifted $t$-sparse which runs in time $d^{O(1)}$ (trying successively $t=1,2, \ldots$ ), moreover this algorithm finds all $Y=Y_{0}$ such that $g\left(X-Y_{0}\right)$ is $t$-sparse. Namely, one writes down a polynomial system in $Y$ equating to zero all the coefficients in the powers of $X$, thus the system contains $d^{O(1)}$ equations of degrees at most $d^{O(1)}$. So, one can prove the following proposition.

Proposition. There is an algorithm which for one-variable polynomial g finds the minimal $t$ and all $Y_{0}$ for which $g\left(X-Y_{0}\right)$ is t-sparse in time $(M d)^{O(1)}$. The number of such $Y_{0}$ does not exceed $d^{O(1)}$.

One of the purposes of the sparse analysis is to get rid of $d$ in the complexity bounds. We can write down a system in $b$ with a less (for small $t$ ) number of equations, when $b$ is supposed to belong to $\mathbb{R}$. So, assume that the expansion $g=\sum_{j} \beta_{j}(X+b)^{j}$ contains at most $t$ terms for some $b \in \mathbb{R}$. Then for any fixed $Y=Y_{0} \in \mathbb{R}$ a polynomial $\left(D_{1}^{K} g\right)\left(X, Y_{0}\right)=\sum_{j} \beta_{j}\left(p_{1}^{K}\left(X+Y_{0}\right)-Y_{0}+b\right)^{j}$ for $K \geq 0$. Therefore the polynomial $\mathcal{W}_{g}\left(X, Y_{0}\right)$ has at most $2^{O\left(t^{4}\right)}$ real roots because of [Kh 91] since one can consider $(2 t+1) t$ powers of linear polynomials $\left(p_{1}^{K}\left(X+Y_{0}\right)-Y_{0}+b\right)^{j}, \quad 0 \leq K \leq 2 t$ as the elements of a Pfaffian chain [Kh 91]. 
Thus $Y$ satisfies the conditions of lemma 3 if and only if it satisfies the following system of polynomial equations (cf. lemma 5 below)

$$
\mathcal{W}_{g}(0, Y)=\mathcal{W}_{g}(1, Y)=\ldots=\mathcal{W}_{g}\left(2^{O\left(t^{4}\right)}, Y\right)=0
$$

Each of the polynomials from the latter system can be represented by a blackbox for its evaluation. As each of these polynomials $\mathcal{W}_{g}(s, Y)$ contains $(2 t+1) t$ powers $\left(p_{1}^{K}(s+Y)-Y+b\right)^{j}, \quad 0 \leq K \leq 2 t$ the system has at most $2^{O\left(t^{4}\right)}$ real solutions (by the same argument relying on [Kh 91] as above), thus the number of such $Y=Y_{0}$ that $g\left(X-Y_{0}\right)$ is $t$-sparse is less than $2^{O\left(t^{4}\right)}$.

\section{Multivariate Shifted Sparse Polynomials}

Consider now $n^{2}+n$ new variables $Z_{i, j}, Y_{i}, \quad 1 \leq i, j \leq n$ and a $\mathbb{Q}\left(\left\{Z_{i j}, Y_{i}\right\}_{1 \leq i, j \leq n}\right)$ linear transformation $D_{n}$ of the ring $\mathbb{Q}\left(\left\{Z_{i j}, \bar{Y}_{i}\right\}_{1 \leq i, j \leq n}\right)\left[X_{1}, \ldots, X_{n}\right]$ mapping

$$
D_{n} X=Z P Z^{-1}(X-Y)+Y
$$

where vectors $X=\left(X_{1}, \ldots, X_{n}\right)^{T}, Y=\left(Y_{1}, \ldots, Y_{n}\right)^{T}$, matrices $Z=\left(Z_{i j}\right), P=$ $\left(\begin{array}{ccc}p_{1} & & 0 \\ & \ddots & \\ 0 & & p_{n}\end{array}\right)$. Similarly, as above denote
\[ \mathcal{W}_{g}(X, Y, Z)=\operatorname{det}\left(\begin{array}{llll}g & D_{n} g & \ldots & D_{n}^{t} g \\ \vdots & \vdots & \\ D_{n}^{t} g & D_{n}^{t+1} g & \ldots & D_{n}^{2 t} g\end{array}\right) \in \mathbb{Q}(Z)[X, Y] . \]

Lemma 4. $g$ is shifted $t$-sparse if and only if for some $Z_{0}, Y_{0}$ such that det $Z_{0} \neq$ 0 , the polynomial $\mathcal{W}_{g}\left(X, Y_{0}, Z_{0}\right)$ vanishes identically. Moreover, in this case a polynomial $g\left(Z_{0} X+Y_{0}\right)$ is t-sparse.

The proof is similar to the proof of lemma 3 taking into account that

$$
\left(D_{n} g\right)(Z X+Y)=g\left(Z P Z^{-1}(Z X+Y-Y)+Y\right)=g(Z P X+Y) .
$$

As in section 2 lemma 4 provides a test for minimal shifted $t$-sparsity trying successively $t=1,2, \ldots$ running in time $d^{O\left(n^{4}\right)}$ (see [CG 83] for solving system of polynomial equations and inequalities). Moreover, the algorithm finds algebraic conditions (equations and inequality $\operatorname{det} Z \neq 0$ ) on all $Z, Y$ for which $g(Z X+Y)$ is $t$-sparse.

So, these $Z, Y$ form a constructive set $U \subset \overline{\mathbb{Q}}^{n^{2}+n}$ given by a system $h_{1}=$ $\ldots=h_{k}=0$, det $Z \neq 0$ where $h_{1}, \ldots, h_{k} \in \mathbb{Q}\left[\left\{Z_{i j}, Y_{i}\right\}_{1<i, j \leq n}\right]$, then $\operatorname{deg}\left(h_{1}\right), \ldots, \operatorname{deg}\left(h_{k}\right) \leq d^{O(1)}, k \leq d^{O(1)}$. Applying the algorithm from [CG 83] one can find the irreducible over $\mathbb{Q}$ components $\bar{U}=\bigcup_{l} U^{(l)}$ of the closure (in the Zariski topology) $\bar{U}$. For each component $U^{(l)}$ the algorithm from [CG 83] produces firstly, some polynomials $h_{1}^{(l)}, \ldots, h_{N(l)}^{(l)} \in \mathbb{Q}\left[\left\{Z_{i j}, Y_{i}\right\}\right]$ such that $U^{(l)}=$ 
$\left\{h_{1}^{(l)}=\ldots=h_{N(l)}^{(l)}=0\right\}$ and secondly, a general point of $U^{(l)}$, namely the following fields isomorphism

$$
\mathbb{Q}\left(U^{(l)}\right) \simeq \mathbb{Q}\left(T_{1}, \ldots, T_{m}\right)[\theta]
$$

where $\mathbb{Q}\left(U^{(l)}\right)$ is the field of rational functions on $U^{(l)}, m=\operatorname{dim}\left(U^{(l)}\right)$, linear forms $T_{1}, \ldots, T_{m}$ in variables $\left\{Z_{i j}, Y_{i}\right\}_{1<i, j<n}$ constitute a transcendental basis of $\mathbb{Q}\left(U^{(l)}\right)$ and $\theta$ is algebraic over $\mathbb{Q}\left(T_{1}, \ldots, T_{m}\right)$. The algorithm produces a minimal polynomial $\phi(Z) \in \mathbb{Q}\left(T_{1}, \ldots, T_{m}\right)[Z]$ of $\theta$, the linear forms $T_{S}\left(\left\{Z_{i j}, Y_{i}\right\}\right), 1 \leq S \leq m$, a linear form $\theta\left(\left\{Z_{i j}, Y_{i}\right\}\right)$, and the expressions for the coordinate functions $Z_{i, j}\left(T_{1}, \ldots, T_{m}, \theta\right), Y_{i}\left(T_{1}, \ldots, T_{m}, \theta\right)$ as rational functions in $T_{1}, \ldots, T_{m}, \theta$. The degrees of the polynomials $h_{1}^{(l)}, \ldots, h_{N(l)}^{(l)}$ do not exceed $d^{O\left(n^{2}\right)}$, the bit-size of any of the (rational) coefficients occuring in these polynomials can be bounded by $M^{O(1)} d^{O\left(n^{2}\right)}$ and the algorithm runs in time $M^{O(1)} d^{O\left(n^{4}\right)}$.

Denote $\tilde{U}^{(l)}=U^{(l)} \backslash\{\operatorname{det} Z=0\}$ (some of $\tilde{U}^{(l)}$ can be empty), remark that $U=\bigcup_{l} \tilde{U}^{(l)}$.

For any point $\left(Z_{0}, Y_{0}\right) \in \tilde{U}^{(l)}$ the polynomial $g\left(Z_{0} X+Y_{0}\right)$ is exactly $t$-sparse, therefore by lemma 2 the following linear system

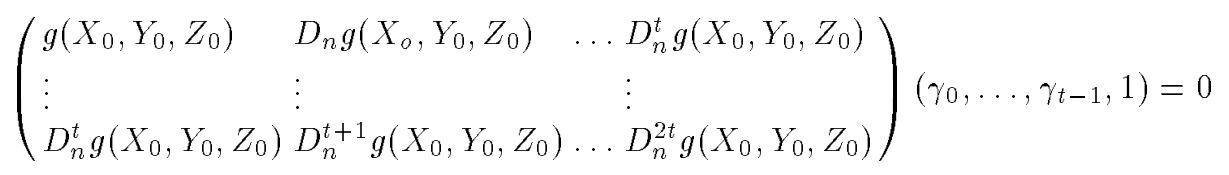

has a unique solution, where the vector $X_{0}=Z_{0}^{-1}\left((1, \ldots, 1)^{T}-Y_{0}\right)$. As $\gamma_{0}, \ldots, \gamma_{t-1} \in \mathbb{Z}$ (see section 1 ) and $\gamma_{0}, \ldots, \gamma_{t-1}$ can be represented as the rational functions in $(Z, Y) \in \tilde{U}^{(l)}$, we conclude taking into account the irreducibility of $U^{(l)}$ that $\gamma_{0}, \ldots, \gamma_{t-1}$ are constants on $\tilde{U}^{(l)}$. Thus, the exponent vectors $I$ (see section 1) are the same for all the points $(Z, Y) \in \tilde{U}^{(l)}$.

So, for $(Z, Y) \in \tilde{U}^{(l)}$ one can write $t$-sparse representation of the polynomial

$$
g=\sum_{I} C_{I}(Z, Y)\left(Z^{-1}(X-Y)\right)^{I}
$$

where the coefficients $C_{I}(Z, Y)$ depend on $Z, Y$. The equality (1) is equivalent to a system of equalities

$$
g\left(Z X^{(0)}+Y\right)=\sum_{I} C_{I}(Z, Y)\left(Z^{-1}\left(X^{(0)}-Y\right)\right)^{I}
$$

where $X^{(0)}$ runs over all the vectors from $\{0, \ldots, d\}^{n}$. Adding to the latter system the system $\operatorname{det} Z \neq 0, h_{1}^{(l)}=\ldots=h_{N(l)}^{(l)}=0$ determining $\tilde{U}^{(l)}$ we come to a parametrical (with the parameters $\left\{Z_{i j}, Y_{i}\right\}$ ) linear in $C_{I}$ system which one can solve invoking the algorithm from [H 83] (see also [CG 84]) in time $M^{O(1)} d^{O\left(n^{4}\right)}$. This algorithm yields some disjoint decomposition of $\tilde{U}^{(l)}=\bigcup_{S} U_{S}^{(l)}$ where each $U_{S}^{(l)}$ is a constructive set and also yields the rational functions $\bar{C}_{I, S}^{(l)}\left(\left\{Z_{i j}, Y_{i}\right\}\right) \in$ 
$\mathbb{Q}\left(\left\{Z_{i j}, Y_{i}\right\}\right)$ such that $C_{I}=\bar{C}_{I, S}^{(l)}\left(\left\{Z_{i j}, Y_{i}\right\}\right)$ for every point $\left\{Z_{i j}, Y_{i}\right\} \in U_{S}^{(l)}$ (thus each $C_{I}$ is a piecewise-rational function on $\left.\tilde{U}^{(l)}\right)$.

The algorithm yields also polynomials $h_{S, 0}^{(l)}, \ldots, h_{S, N_{S}^{(l)}}^{(l)} \in \mathbb{Q}\left[\left\{Z_{i j}, Y_{i}\right\}\right]$ such that $U_{S}^{(l)}=\left\{h_{S, 0}^{(l)} \neq 0, h_{S, 1}^{(l)}=\ldots=h_{S, N_{S}^{(l)}}^{(l)}=0\right\}$. iFrom [H 83] (see also [CG 84]) we get the bounds on the degrees $\operatorname{deg}\left(h_{S, q}^{(l)}\right), \operatorname{deg}\left(\bar{C}_{I, S}^{(l)}\right) \leq d^{O\left(n^{2}\right)}$ and the bound $M^{O(1)} d^{O\left(n^{2}\right)}$ for the bit-size of every (rational) coefficients of all the yielded rational functions.

Thus, we have proved the following theorem (cf. proposition above).

Theorem. There is an algorithm which finds a minimal t and produces a constructive set $U \subset \overline{\mathbb{Q}}^{n^{2}+n}$ of all $\left\{Z_{i j}, Y_{i}\right\}_{1<i, j<n}$ such that $g(Z X+Y)$ is t-sparse, in the form $U=\bigcup_{l} \mathcal{U}^{(l)}$ and for each constructive set $\mathcal{U}^{(l)}$ the algorithm produces polynomials $\mathcal{H}_{0}^{(l)}, \ldots, \mathcal{H}_{\mathcal{N}^{(l)}}^{(l)} \in \mathbb{Q}\left[\left\{Z_{i j}, Y_{i}\right\}\right]$ such that $\mathcal{U}^{(l)}=\left\{\mathcal{H}_{0}^{(l)} \neq\right.$ $\left.0, \mathcal{H}_{1}^{(l)}=\ldots=\mathcal{H}_{\mathcal{N}^{(l)}}^{(l)}=0\right\}$. Also the algorithm produces $t$ exponent vectors and for each exponent vector I a rational function $\mathcal{C}_{I}^{(l)}\left(\left\{Z_{i j}, Y_{i}\right\}\right) \in \mathbb{Q}\left(\left\{Z_{i j}, Y_{i}\right\}\right)$ which provide t-sparse representations of

$$
g=\sum_{I} \mathcal{C}_{I}^{(l)}\left(\left\{Z_{i j}, Y_{i}\right\}\right)\left(Z^{-1}(X-Y)\right)^{I}
$$

which is valid for every point $\left(\left\{Z_{i j}, Y_{i}\right\}\right) \in \mathcal{U}^{(l)}$. The degrees of all produced rational functions $\mathcal{H}_{S}^{(l)}, \mathcal{C}_{I}^{(l)}$ do not exceed $d^{O\left(n^{2}\right)}$, the bit-size of the coefficients of these rational functions can be bounded by $\left(M d^{n^{2}}\right)^{O(1)}$ and the running time of the algorithm is at most $\left(M d^{n^{4}}\right)^{O(1)}$.

Again when $Z_{i j}, Y_{i}$ belong to $\mathbb{R}$ we could write down a polynomial system on $Z, Y$ with a less number of equations. For this purpose we need the following

Lemma 5. If $g$ is a shifted t-sparse polynomial, then for any $Z_{0}, Y_{0}$ such that $\operatorname{det} Z_{0} \neq 0$ for at least one of $X_{1}^{(0)}=1, \ldots, n^{O(n)} 2^{O\left(t^{4}\right)}$, a polynomial $\mathcal{W}_{g}\left(X_{1}^{(0)}, X_{2}, \ldots, X_{n}, Y_{0}, Z_{0}\right) \in \mathbb{R}\left[X_{2}, \ldots, X_{n}\right]$ does not vanish identically, provided that $\mathcal{W}_{g}\left(X, Y_{0}, Z_{0}\right) \in \mathbb{R}[X]$ does not vanish identically.

Proof. Let for some $Z^{(0)}, Y^{(0)}$ a polynomial $g\left(Z^{(0)} X+Y^{(0)}\right)$ be $t$-sparse, i.e.

$$
g=\sum_{J} \beta_{J} \prod_{1 \leq i \leq n}\left(\left(Z^{(0)}\right)^{-1}\left(X-Y^{(0)}\right)\right)_{i}^{j_{i}}
$$

where $J=\left(j_{1}, \ldots, j_{n}\right)$ and the sum has at most $t$ items $\left(\right.$ by $\left(\left(Z^{(0)}\right)^{-1}\left(X-Y^{(0)}\right)\right)_{i}$ we denote $i$-th coordinate of the vector $\left.\left(Z^{(0)}\right)^{-1}\left(X-Y^{(0)}\right)\right)$. Then

$\left(D_{n}^{K} g\right)\left(X, Y_{0}, Z_{0}\right)=\sum_{J} \beta_{J} \prod_{1 \leq i \leq n}\left(\left(Z^{(0)}\right)^{-1}\left(\left(Z_{0} P^{K} Z_{0}^{-1}\left(X-Y_{0}\right)+Y_{0}\right)-Y^{(0)}\right)\right)_{i}^{j_{i}} \quad$ for $0 \leq K \leq 2 t$.

Thus $\mathcal{W}_{g}\left(X, Y_{0}, Z_{0}\right)$ is a polynomial in $(2 t+1) t$ products of the form like in the latter expression and these products can be considered as the elements of a Pfaffian chain. [Kh 91] entails (cf. also [GKS 93]) that the sum of Betti numbers 
of the variety $\left\{\mathcal{W}_{g}\left(X, Y_{0}, Z_{0}\right)=0\right\} \subset \mathbb{R}^{n}$ is less than $n^{O(n)} 2^{O\left(t^{4}\right)}$. As in particular $(n-1)$-th Betti number $b^{n-1}<n^{O(n)} 2^{O\left(t^{4}\right)}$ we conclude the statement of the lemma (cf. [GKS 93]).

Thus, $Y, Z$ satisfy the conditions of lemma 4 if and only if $\operatorname{det} Z \neq 0$ and they satisfy the following $n^{O\left(n^{2}\right)} 2^{O\left(n t^{4}\right)}$ equations.

$$
\mathcal{W}_{g}\left(X_{1}^{(0)}, \ldots, X_{n}^{(0)}, Y, Z\right)=0, \quad X_{1}^{(0)}, \ldots, X_{n}^{(0)} \in\left\{1, \ldots, n^{O(n)} 2^{O\left(t^{4}\right)}\right\}
$$

\section{Zero-test for shifted sparse polynomials}

Let $g$ be shifted $t$-sparse polynomial. Then (see lemma 5 ) for at least one of $X_{1}^{(0)}=1, \ldots, n^{O(n)} 2^{\left(t^{2}\right)}$ a polynomial $g\left(X_{1}^{(0)}, X_{2}, \ldots, X_{n}\right) \in \mathbb{Q}\left[X_{2}, \ldots, X_{n}\right]$ does not vanish identically. Thus for zero-test one can compute $g\left(X_{1}^{(0)}, \ldots, X_{n}^{(0)}\right)$ for $n^{O\left(n^{2}\right)} 2^{O\left(n t^{2}\right)}$ points $\left(X_{1}^{(0)}, \ldots, X_{n}^{(0)}\right) \in\left\{1, \ldots, n^{O(n)} 2_{2} O\left(t^{2}\right)\right\}^{n}$. Then $g$ vanishes identically if and only if all the results of computation vanish. Thus, the complexity of zero-test does not depend on $d$.

Acknowledgement. The authors would like to thank C. Schnorr for initiating the question about the shifted sparse polynomials.

\section{References}

[BT 88] Ben-Or, M. \& Tiwari, P., A deterministic algorithm for sparse multivariate polynomial interpolation, Proc. 20 STOC ACM, 1988, pp. 301309.

[CG 83] Chistov, A. \& Grigoriev, D., Subexponential-time solving systems of algebraic equations, Preprints LOMI E-9-83, E-10-83, Leningrad, 1983.

[CG 84] Chistov, A. \& Grigoriev, D., Complexity of quantifier elimination in the theory of algebraically closed fields, Lect. Notes Comp. Sci. 176, 1984, pp. 17-31.

[GK 87] Grigoriev, D. \& Karpinski, M., The matching problem for bipartite graphs with polynomially bounded permanents is in NC, Proc. 28 FOCS IEEE, 1987, pp. 166-172.

[GKS 90] Grigoriev, D., Karpinski, M. \& Singer, M., Fast parallel algorithms for sparse multivariate polynimial interpolation over finite fields, SIAM J. Comput. 19, N 6, 1990, pp. 1059-1063.

[GKS 91] Grigoriev, D., Karpinski, M. \& Singer, M., The interpolation problem for k-sparse sums of eigenfunctions of operators, Adv. Appl. Math. 12, 1991, pp. 76-81.

[GKS 92] Grigoriev, D., Karpinski, M. \& Singer, M., Computational complexity of sparse rational interpolation, to appear in SIAM J. Comput.

[GKS 93] Grigoriev, D., Karpinski, M. \& Singer, M., Computational complexity of sparse real algebraic function interpolation, to appear in Proc. Int. Conf. Eff. Meth. Alg. Geom., Nice, April 1992 (Progr. in Math. Birkhäuser).

[H 83] Heintz, J., Definability and fast quantifier elimination in algebraically closed fields, Theor. Comp. Sci. 24, 1983, pp. 239-278. 
[Ka 89] Karpinski, M., Boolean Circuit Complexity of Algebraic Interpolation Problems, Technical Report TR-89-027, International Computer Science Institute, Berkeley, 1989; in Proc. CSL'88, Lecture Notes in Computer Science 385, 1989, pp. 138-147.

[Kh 91] Khovanski, A., Fewnomials, Transl. Math. Monogr., AMS 88, 1991.

[KY 88] Kaltofen, E. \& Yagati, L., Improved sparse multivariate interpolation, Report 88-17, Dept. Comput. Sci., Rensselaer Polytechnic Institute, 1988.

This article was processed using the $\mathrm{IAT}_{\mathrm{E}} \mathrm{X}$ macro package with LLNCS style 